Pacific Journal of Mathematics

BANACH ALGEBRAS OF LIPCHITZ FUNCTIONS 


\section{BANACH ALGEBRAS OF LIPSCHITZ FUNCTIONS}

\section{DONALD R. SHeRberT}

1. Lip $(X, d)$ will denote the collection of all bounded complexvalued functions defined on the metric space $(X, d)$ that satisfy a Lipschitz condition with respect to the metric $d$. That is, $\operatorname{Lip}(X, d)$ consists of all $f$ defined on $X$ such that both

$$
\|f\|_{\infty}=\sup \{|f(x)|: x \in X\}
$$

and

$$
\|f\|_{a}=\sup \{|f(x)-f(y)| / d(x, y): x, y \in X, x \neq y\}
$$

are finite. With the norm $\|\cdot\|$ defined by $\|f\|=\|f\|_{\infty}+\|f\|_{d}, \operatorname{Lip}(X, d)$ is a Banach algebra. We shall sometimes refer to such an algebra as a Lipschitz algebra. In this paper we investigate some of the basic properties of these Banach algebras.

It will be assumed throughout the paper that $(X, d)$ is a complete metric space. There is no loss of generality in doing so: for suppose $(X, d)$ were not complete and let $\left(X^{\prime}, d^{\prime}\right)$ denote its completion. Since each element of Lip $(X, d)$ is uniformly continuous on $(X, d)$, it extends uniquely and in a norm preserving way to an element of $\operatorname{Lip}\left(X^{\prime}, d^{\prime}\right)$. Thus as Banach algebras, $\operatorname{Lip}(X, d)$ and $\operatorname{Lip}\left(X^{\prime}, d^{\prime}\right)$ are isometrically isomorphic.

In $\S 2$ we sketch briefly the main points of the Gelfand theory and observe that every commutative semi-simple Banach algebra $A$ is isomorphic to a subalgebra of the Lipschitz algebra $\operatorname{Lip}(\Sigma, \sigma)$, where $\Sigma$ is the carrier space of $A$ and $\sigma$ is the metric $\Sigma$ inherits from being a subset of the dual space $A^{*}$ of $A$. This representation is obtained from the Gelfand representation; instead of using the usual Gelfand (relative weak*) topology of $\Sigma$, the metric topology is used. Later, in $\S 4$, we show that this isomorphism is onto if and only if $A=\operatorname{Lip}(X, d)$ for a compact $(X, d)$.

In $\S 3$ we study the carrier space $\Sigma$ of $\operatorname{Lip}(X, d)$. The fact that $\operatorname{Lip}(X, d)$ is a point separating algebra of functions on $X$ allows us to identify $X$ as a subset of $\Sigma$. The topologies $X$ inherits from $\Sigma$ are compared to the original $d$-topology; they are shown to be equivalent and in the case of the two metric topologies we show them to be equivalent in a strong sense. In Theorem 3.9 we show that the important case of $\Sigma=X$ is equivalent to $(X, d)$ being compact,

Received November 14, 1962. This paper is based on a portion of the author's $\mathrm{Ph}$. D. dissertation written at Stanford University under the supervision of Professor Karel de Leeuw. 
and also equivalent to $(\Sigma, \sigma)$ being compact.

The Gelfand representation for $\operatorname{Lip}(X, d)$ is considered in $\S 4$. The image of Lip $(X, d)$ under the Gelfand mapping turns out to be precisely those functions in $\operatorname{Lip}(\Sigma, \sigma)$ that are continuous on $\Sigma$ in the Gelfand topology.

In $\S 5$ we identify the homomorphisms from $\operatorname{Lip}\left(X_{1}, d_{1}\right)$ into Lip $\left(X_{2}, d_{2}\right)$ where the $\left(X_{i}, d_{i}\right)$ are compact. As a corollary the automorphisms of $\operatorname{Lip}(X, d)$ for compact $(X, d)$ are obtained.

2. Let $A$ be a semi-simple commutative Benach algebra with identity and with norm $\|\cdot\|_{A}$. The collection of nonzero multiplicative linear functionals on $A$ is called the carrier space of $A$ and will be denoted by $\Sigma$. It is well known [1, p. 69] that these functionals are bounded so that $\Sigma$ forms a subset of the dual space $A^{*}$ of $A$. In fact, $\Sigma$ lies on the unit sphere of $A^{*}$. As a subset of $A^{*}$ the carrier space $\Sigma$ inherits two important topologies : the relative weak* topology, which we shall refer to as the Gelfand topology of $\Sigma$, and the relative norm, or metric topology.

The Gelfand theory of commutative Banach algebras utilizes the former topology. When $A$ has an identity, $\Sigma$ with its Gelfand topology is compact. For each $f \in A$ the function $\hat{f}$ is defined on $\Sigma$ by $\hat{f}(\varphi)=$ $\varphi f, \varphi \in \Sigma$. Each $\hat{f}$ is continuous on $\Sigma$ in the Gelfand topology; indeed, the Gelfand topology is precisely the weakest topology on $\Sigma$ such. that the family $\{\hat{f}: f \in A\}$ is continuous. Let $C(\Sigma)$ denote the space. of complex-valued functions on $\Sigma$ continuous in the Gelfand topology supplied with sup norm $\|\cdot\|_{\infty}$. Then the Gelfand mapping $f \rightarrow \hat{f}$ is. an isomorphism of $A$ into $C(\Sigma)$ and is norm decreasing. Details of the Gelfand theory can be found in $[1,2]$.

Let us now consider the metric topology of $\Sigma$. The norm $\|\cdot\|_{A}^{*}$ of the dual space $A^{*}$ is defined by

$$
\|\varphi\|_{A}^{*}=\sup \left\{|\varphi f|: f \in A,\|f\|_{A} \leqq 1\right\} \quad \varphi \in A^{*} .
$$

The metric $\sigma$ on $\Sigma$ induced by this norm is given by

$$
\sigma(\varphi, \psi)=\|\varphi-\psi\|_{A}^{*} \quad \varphi, \psi \in \Sigma .
$$

In terms of the functions $\hat{f}, f \in A$, we may express the metric $\sigma$ by

$$
\sigma(\varphi, \psi)=\sup \left\{|\hat{f}(\varphi)-\hat{f}(\psi)|: f \in A,\|f\|_{A} \leqq 1\right\} \quad \varphi, \psi \in \Sigma .
$$

The metric topology of $\Sigma$ is stronger than the Gelfand topology. Therefore, since $\Sigma$ is closed in $A^{*}$ in the weak ${ }^{*}$ topology, it is also. closed in the metric topology. Hence, $(\Sigma, \sigma)$ is a complete metric space. Also each $\hat{f}, f \in A$, is continuous on $(\Sigma, \sigma)$ since it is continuous on $\Sigma$ in the Gelfand topology. The metric $\sigma$ is bounded. 
because $\Sigma$ lies on the unit sphere of $A^{*}$.

With this metric space $(\Sigma, \sigma)$ we can form the Lipschitz algebra. $\operatorname{Lip}(\Sigma, \sigma)$ with norm $\|\cdot\|$ given by

$$
\|g\|=\|g\|_{\infty}+\|g\|_{\sigma} \quad g \in \operatorname{Lip}(\Sigma, \sigma) .
$$

We now show that the Gelfand mapping takes $A$ into this Lipschitz algebra to yield what might be termed a "Lipschitz representation".

Proposition 2.1. Let $A$ be a semi-simple commutative Banach algebra with identity. Then the Gelfand mapping is a continuous isomorphism of $A$ onto a subalgebra of $\operatorname{Lip}(\Sigma, \sigma)$. Furthermore, for each $f \in A$,

$$
\|\hat{f}\|_{\sigma} \leqq\|f\|_{A} \text { and }\|\hat{f}\|_{\infty} \leqq\|f\|_{A} \text {. }
$$

Proof. For $g \in A$ with $\|g\|_{A} \leqq 1$ we have from (2.1) that

$$
|g(\varphi)-g(\psi)| \leqq \sigma(\varphi, \psi) \quad \varphi, \psi \in \Sigma .
$$

Then for any nonzero $f \in A$, the element $\frac{1}{\|f\|_{A}} f$ has norm 1 , so that from (2.2) we obtain

$$
|\hat{f}(\varphi)-\hat{f}(\psi)| \leqq\|f\|_{A} \sigma(\varphi, \psi) \quad \varphi, \psi \in \Sigma .
$$

Hence, $\|\hat{f}\|_{\sigma} \leqq\|f\|_{A}$, for all $f \in A$. From the Gelfand theory we have $\|\hat{f}\|_{\infty} \leqq\|f\|_{A}$, all $f \in A$. Thus, the mapping $f \rightarrow \hat{f}$ takes $A$ continuously into $\operatorname{Lip}(\Sigma, \sigma)$. From the Gelfand theory we know that. the mapping is an isomorphism, hence the image of $A$ is a subalgebra. of $\operatorname{Lip}(\Sigma, \sigma)$.

In $\S 4$ we consider the Gelfand mapping for the case $A=\operatorname{Lip}(X, d)$.

3. The Banach algebra $\operatorname{Lip}(X, d)$ is an algebra of functions defined on a set $X$. The function identically 1 is its identity and it is evidently a self-adjoint (closed under complex conjugation) algebra. We now observe that it separates the points of $X$.

For fixed $s \in X$ define the function $f$ on $X$ by $f(x)=d(x, s), x \in X$. An application of the triangle inequality for $d$ shows that $\|f\|_{d} \leqq 1$. However, if the metric is unbounded, then the function $f$ so defined is not an element of $\operatorname{Lip}(X, d)$. This is remedied by truncation. The following lemma is easily verified.

Lemma 3.1. For each $s \in X$, the function $f_{s}$ defined on $X$ by

$$
f_{s}(x)=\min \{d(x, s), 1\} \quad x \in X
$$

belongs to $\operatorname{Lip}(X, d)$ and has norm $\left\|f_{s}\right\| \leqq 2$. The family of functions $\left\{f_{s}: s \in X\right\}$ separates the points of $X$. 
Let $\Sigma$ denote the carrier space of $\operatorname{Lip}(X, d)$. Since $\operatorname{Lip}(X, d)$ is a point separating algebra of functions on $X$ each $x \in X$ can be identified with the evaluation functional $\varphi_{x}$ in $\Sigma$ where $\varphi_{x}(f)=f(x)$. More precisely, since the algebra separates points the injection mapping $x \rightarrow \varphi_{x}$ is one-to-one from $X$ to $\Sigma$. Thus we may regard $X$ as a subset of $\Sigma$.

An algebra of functions defined on a set $X$ is called inverseclosed if for every function $f$ in the algebra satisfying $|f(x)| \geqq \varepsilon>0$, all $x \in X$, the inverse $f^{-1}$ is also in the algebra. It is not difficult to check that $\operatorname{Lip}(X, d)$ is an inverse-closed algebra.

The next lemma is a consequence of the general theory of function algebras and holds for any algebra of functions on a set that is self-adjoint, separates points and is inverse-closed. See [1, p. 55].

LEMMA 3.2. Let $\Sigma$ be the carrier space of $\operatorname{Lip}(X, d)$. Then $X$ is dense in $\Sigma$ in the Gelfand topology. If $(X, d)$ is compact, then $X=\Sigma$ and the Gelfand topology coincides with the d-topology of $X$.

As a subset of $\Sigma, X$ inherits two more topologies-the Gelfand and the metric topologies of $\Sigma$. The remainder of this section is concerned with the comparison of these inherited topologies of $X$ to its original $d$-topology. We first look at the relative Gelfand topology of $X$. A basic neighborhood of $x_{0} \in X$ in the relative Gelfand topology is of the form

$$
\begin{aligned}
& N\left(x_{0}, f_{1}, \cdots, f_{n}, \varepsilon\right) \\
& \quad=\left\{x \in X:\left|f_{i}(x)-f_{i}\left(x_{0}\right)\right|<\varepsilon, i=1,2, \cdots, n\right\}
\end{aligned}
$$

where the $f_{i}$ are elements of $\operatorname{Lip}(X, d)$ and $\varepsilon$ is a positive number.

Proposition 3.3. The relative Gelfand topology of $X$ and the d-topology of $X$ are equivalent.

Proof. It is clear from the definition of the Gelfand topology that the relative Gelfand topology of $X$ is weaker than the $d$-topology. To show that every $d$-open set of $X$ is also open in the relative Gelfand topology, it suffices to show that given $x_{0} \in X$ and $\varepsilon>0$ the sphere $S\left(x_{0}, \varepsilon\right)=\left\{x \in X: d\left(x, x_{0}\right)<\varepsilon\right\}$ is open in the relative Gelfand topology. Define the function $f$ on $X$ by (3.1) for $s=x_{0}$.

Then $f$ belongs to $\operatorname{Lip}(X, d)$ and the neighborhood of $x_{0}$ in the relative Gelfand topology determined by $f$ and $\varepsilon$ is precisely (we assume $\varepsilon<1$ )

$$
N\left(x_{0}, f, \varepsilon\right)=\left\{x \in X:\left|f(x)-f\left(x_{0}\right)\right|<\varepsilon\right\}=S\left(x_{0}, \varepsilon\right) .
$$


Hence, the spheres $S\left(x_{0}, \varepsilon\right)$ are open in the relative Gelfand topology and the proof is finished.

We now turn to the comparison of the two metric topologies on $X$. The metric $\sigma$ on $\Sigma$ is defined by (2.1). When restricted to the subset $X$ of $\Sigma$, the metric $\sigma$ can be expressed by

$$
\sigma(x, y)=\sup \{|f(x)-f(y)|: f \in \operatorname{Lip}(X, d),\|f\| \leqq 1\} \quad x, y \in X .
$$

The next few propositions are concerned with the relation between $d$ and $\sigma$ on $X$. We first define the notions of equivalence of metrics which will be the pertinent ones in this discussion.

Definition. Two metrics $d_{1}$ and $d_{2}$ on a space $X$ are called boundedly equivalent if and only if there exist positive numbers $K_{1}$ and $K_{2}$ such that

$$
K_{1} d_{1}(x, y) \leqq d_{2}(x, y) \leqq K_{2} d_{1}(x, y) \quad x, y \in X .
$$

They are called uniformly equivalent if and only if the identity mapping from $\left(X, d_{1}\right)$ to $\left(X, d_{2}\right)$ is a uniform homeomorphism.

Bounded equivalence implies uniform equivalence, but not conversely. For example, the metrics $d_{1}(x, y)=|x-y|$ and $d_{2}(x, y)=$ $|x-y|^{1 / 2}$ on $[0,1]$ are uniformly but not boundedly equivalent. An unbounded metric can never be boundedly equivalent to a bounded metric. Therefore if $d$ is an unbounded metric it can not be boundedly equivalent to the metric $d /(1+d)$ although it is well know that these two metrics are uniformly equivalent.

Since $\Sigma$ lies on the unit sphere of the dual space of $\operatorname{Lip}(X, d)$, the diameter of $(\Sigma, \sigma)$ is at most two. Thus $\sigma$ is always a bounded metric. If the original metric $d$ on $X$ is boundedly equivalent to the inherited metric $\sigma$, then $d$ must also be a bounded metric. It turns out that the converse is also true.

Proposition 3.4. If the diameter of $(X, d)$ is finite, then the metric $\sigma$ on $X$ defined by (3.2) is boundedly equivalent to $d$.

Proof. If $f \in \operatorname{Lip}(X, d)$ satisfies $\|f\| \leqq 1$, then $|f(x)-f(y)| \leqq d(x, y)$, all $x, y$ in $X$. Hence, forming the supremum over all $f$ with $\|f\| \leqq 1$, we obtain $\sigma(x, y) \leqq d(x, y)$, all $x, y$ in $X$.

Let $D$ denote the diameter of $(X, d)$. Let $x$ and $y$ in $X$ be given. Define $f$ by $f(u)=d(u, x), u \in X$. Then $f \in \operatorname{Lip}(X, d)$ and $\|f\| \leqq 1+D$, so that $g=K f$ where $K=1 /(1+D)$ has norm at most 1. Hence

$$
\sigma(x, y) \geqq|g(x)-g(y)|=K d(x, y) .
$$


Thus for all $x, y$ in $X$ we have

$$
K d(x, y) \leqq \sigma(x, y) \leqq d(x, y)
$$

and the proposition is proved.

In particular, if $(X, d)$ is compact, then the diameter is finite and $\sigma$ and $d$ are boundedly equivalent.

We show next as a corollary that bounded equivalence of metrics on a space $X$ is the appropriate notion when dealing with Lipschitz functions in the sense that two metrics on $X$ yield the same class of Lipschitz functions if and only if the metrics are boundedly equivalent.

COROLlaRY 3.5. Let $d_{1}$ and $d_{2}$ be bounded metrics on $X$. Then $A_{1}=\operatorname{Lip}\left(X, d_{1}\right)$ and $A_{2}=\operatorname{Lip}\left(X, d_{2}\right)$ have the same elements if and only if $d_{1}$ and $d_{2}$ are boundedly equivalent.

Proof. It is clear that bounded equivalence of metrics preserves Lipschitz functions. For the converse, suppose $A_{1}=A_{2}$. By the uniqueness of norm theorem for semisimple commutative Banach algebras [2, p. 75], the norms on $A_{1}=A_{2}$ determined by $d_{1}$ and $d_{2}$ are equivalent. Therefore the norms on the dual space $A_{1}^{*}=A_{2}^{*}$ are equivalent. Then the metrics $\sigma_{i}$ on $\Sigma\left(A_{i}\right), i=1,2$, are boundedly equivalent. Since the $d_{i}$ are bounded metrics it follows from Proposition 3.4 that $d_{i}$ is boundedly equivalent to $\sigma_{i}$ on $X, i=1,2$. The relation of bounded equivalence is evidently transitive. Hence $d_{1}$ is boundedly equivalent to $d_{2}$.

As remarked previously Proposition 3.4 is false for spaces $(X, d)$ of inifinite diameter. But the next proposition shows that from the viewpoint of Banach space theory there is no loss in generality in assuming that $d$ is always a bounded metric. We use the fact that for a given metric $d$ on $X, d /(1+d)$ is also a metric on $X$ and is uniformly equivalent to $d$.

Proposition 3.6. Given the metric space $(X, d)$, the Banach algebras $\operatorname{Lip}(X, d)$ and Lip $\left(X, d^{\prime}\right)$ where $d^{\prime}=d /(1+d)$ have the same elements and their norms are equivalent.

Proof. Let $\|\cdot\|^{\prime}$ denote the norm on $\operatorname{Lip}\left(X, d^{\prime}\right)$. Since $d^{\prime}(x, y) \leqq d(x, y)$ for all $x, y$ in $X$ we have $\|\cdot\|_{a} \leqq\|\cdot\|_{d^{\prime}}$. Sup norms are unaffected by a change of metrics, so we have $\|\cdot\| \leqq\|\cdot\|^{\prime}$. On the other hand, 


$$
\begin{aligned}
\|f\|_{d^{\prime}} & =\sup \left\{\frac{|f(x)-f(y)|}{d(x, y)}(1+d(x, y)): x, y \in X\right\} \\
& =\sup \left\{\frac{|f(x)-f(y)|}{d(x, y)}+|f(x)-f(y)|: x, y \in X\right\} \\
& \leqq \sup \left\{\frac{|f(x)-f(y)|}{d(x, y)}+|f(x)|+|f(y)|: x, y \in X\right\} \\
& \leqq\|f\|_{d}+2\|f\|_{\infty} .
\end{aligned}
$$

Thus $\|\cdot\|^{\prime} \leqq 3\|\cdot\|$. Hence Lipschitz functions are preserved so that. $\operatorname{Lip}(X, d)=\operatorname{Lip}\left(X, d^{\prime}\right)$, the norms are equivalent, and the proof is complete.

CoROLlaRy 3.7. The metrics $d$ and $\sigma$ on $X$ are always uniformly equivalent.

Proof. That the metrics $d$ and $d^{\prime}=d /(1+d)$ are uniformly equivalent is well known. We have shown in Proposition 3.6 that the norms $\|\cdot\|$ and $\|\cdot\|^{\prime}$ determined by $d$ and $d^{\prime}$ respectively are equivalent. Therefore, the corresponding norms on the dual space are equivalent. Thus the metrics $\sigma$ and $\sigma^{\prime}$ on $\Sigma$ corresponding to $d$. and $d^{\prime}$ respectively are boundedly equivalent. Since $d^{\prime}$ is bounded by 1 , the metrics $d^{\prime}$ and $\sigma^{\prime}$ on $X$ are boundedly equivalent by Proposition 3.4. Hence, $d$ is uniformly equivalent to $d^{\prime}$, which is boundedly equivalent to $\sigma^{\prime}$, which in turn is boundedly equivalent to $\sigma$. It. follows that $d$ and $\sigma$ are uniformly equivalent.

We have seen that when $\Sigma$ has its Gelfand topology, $X$ appears as a dense subset. In contrast to this, the following lemma shows that when $\Sigma$ has its metric topology $X$ is a closed subset of $\Sigma$. Note. that the standing hypothesis that $(X, d)$ be complete is used explicitly for the first time.

Lemma 3.8. The subset $X$ of $\Sigma$ is closed in the metric topology of $\Sigma$.

Proof. Let $\left\{x_{n}\right\}$ be a sequence $X$ such that $\sigma\left(x_{n}, \xi\right) \rightarrow 0$ where $\xi \in \Sigma$. We must show that $\xi \in X$. The sequence $\left\{x_{n}\right\}$ is $\sigma$-Cauchy since it converges in $(\Sigma, \sigma)$. Uniform equivalence of metrics preserves Cauchy sequences. Therefore, since $d$ and $\sigma$ are uniformly equivalent on $X$ by Corollary 3.7 and since $\left\{x_{n}\right\} \subset X$, the sequence $\left\{x_{n}\right\}$ is $d$-Cauchy. The completeness of $(X, d)$ then implies that $\lim x_{n}=\xi$ belongs to $X$. Hence, $X$ is closed in $(\Sigma, \sigma)$.

Although certain of the implications in the next theorem have been established, we state them here for the sake of unity. The: 
point of interest here is that the set equality $X=\Sigma$ alone is enough to imply compactness of the spaces under consideration. For an arbitrary Banach algebra of functions defined on a space $X$, the fact that the carrier space is just $X$ does not in general have topological ramifications. In the case of $\operatorname{Lip}(X, d)$, however, we have the following.

THEOREM 3.9. The following statements are equivalent:

(i) $X=\Sigma$

(ii) The Gelfand and metric topologies on $\Sigma$ coincide.

(iii) $(\Sigma, \sigma)$ is compact.

(iv) $(X, d)$ is compact.

Proof. (i) $\rightarrow$ (ii) follows from Proposition 3.3. (ii) $\rightarrow$ (iii) is a triviality. To see (iii) $\rightarrow$ (iv), note that if $(\Sigma, \sigma)$ is compact, then by Lemma $3.8, X$ is a closed, hence compact subset of $(\Sigma, \sigma)$. Since $\sigma$ and $d$ are equivalent on $X$, we have that $(X, d)$ is compact. Finally, (iv) $\rightarrow$ (i) follows from Lemma 3.2.

4. We now turn to the Gelfand representation of $\operatorname{Lip}(X, d)$ The general Gelfand theory was mentioned in $\S 2$. So that no confusion of norms will arise here let the norm in $\operatorname{Lip}(\Sigma, \sigma)$ be denoted by $|1| \cdot \mid \|$; then

$$
\left\||| g||=\left|\|g\|_{\infty}+\||| g\|_{\sigma} \quad g \in \operatorname{Lip}(\Sigma, \sigma)\right.\right.
$$

where $\||g|\|_{\infty}$ and $\||g|\|_{\sigma}$ denote the sup norm and Lipschitz norm respectively of $g$ on $(\Sigma, \sigma)$. Proposition 2.1 tells us that the Gelfand mapping $f \rightarrow \hat{f}$ takes $\operatorname{Lip}(X, d)$ isomorphically into $\operatorname{Lip}(\Sigma, \sigma)$ and satisfies $\mid\|\hat{f}\|_{\infty} \leqq\|f\|$ and $\|\hat{f}\|_{\sigma} \leqq\|f\|$, all $f \in \operatorname{Lip}(X, d)$. These statements followed from general considerations. But in the particular case of $\operatorname{Lip}(X, d)$ this can be strengthened.

THEOREM 4.1. The Gelfand mapping $f \rightarrow \hat{f}$ is an isomorphism of $\operatorname{Lip}(X, d)$ onto the closed subalgebra of Lip $(\Sigma, \sigma)$ consisting of those functions in $\operatorname{Lip}(\Sigma, \sigma)$ that are continuous in the Gelfand topology of $\Sigma$.

Proof. If $f \in \operatorname{Lip}(X, d)$ and $\|f\| \leqq 1$, then $\|f\|_{d} \leqq 1$ so that $|f(x)-f(y)| \leqq d(x, y)$, all $x, y \in X$. Thus we have $\sigma(x, y) \leqq d(x, y)$ for all $x, y$ in $X$. Hence for any $f \in \operatorname{Lip}(X, d)$ 


$$
\begin{aligned}
\|f\|_{a} & =\sup \left\{\frac{|f(x)-f(y)|}{d(x, y)}: x, y \in X\right\} \\
& \leqq \sup \left\{\frac{|f(x)-f(y)|}{\sigma(x, y)}: x, y \in X\right\} \\
& \leqq \sup \left\{\frac{|\hat{f}(\varphi)-\hat{f}(\psi)|}{\sigma(\varphi, \psi)}: \varphi, \psi \in \Sigma\right\} \\
& =\|\hat{f} \mid\|_{\sigma} .
\end{aligned}
$$

Since each $\hat{f}, f \in \operatorname{Lip}(X, d)$, is continuous on $\Sigma$ in the Gelfand topology and since $X$ is dense in $\Sigma$ in the Gelfand topology, we have the sup. norm preserved: $\|f\|_{\infty}=\mid\|\hat{f}\|_{\infty}$. Thus for all $f \in \operatorname{Lip}(X, d)$,

$$
\|f\|=\|f\|_{\infty}+\|f\|_{d} \leqq\|\hat{f}\|_{\infty}+\left|\|\hat{f} \mid\|_{\sigma}=\|\hat{f}\| .\right.
$$

This together with the inequality from Proposition 2.1 yields. $\|f\| \leqq\|\hat{f}\| \mid \leqq 2\|f\|$. Hence the mapping $f \rightarrow \widehat{f}$ is a bicontinuous isomorphism, and the image of $\operatorname{Lip}(X, d)$ is therefore a closed subalgebra of $\operatorname{Lip}(\Sigma, \sigma)$.

Let $g \in \operatorname{Lip}(\Sigma, \sigma)$ be continuous on $\Sigma$ in the Gelfand topology; let $f=g \mid X$ denote the restriction of $g$ to $X$. Then $f \in \operatorname{Lip}(X, d)$, since $\sigma(x, y) \leqq d(x, y)$ for all $x, y$ in $X$; and $\hat{f}=g$, since both are continuous on $\Sigma$ in the Gelfand topology and agree on the dense subset $X$. Thus those $g \in \operatorname{Lip}(\Sigma, \sigma)$ which are continuous in the Gelfand topology lie in the range of the mapping $f \rightarrow \widehat{f}$ from $\operatorname{Lip}(X, d)$. Since every $\hat{f}$ is continuous on $\Sigma$ in the Gelfand topology, we see that the image of $\operatorname{Lip}(X, d)$ under the mapping $f \rightarrow \hat{f}$ is exactly the set of functions in $\operatorname{Lip}(\Sigma, \sigma)$ which are continuous on $\Sigma$ in the Gelfand topology. This completes the proof.

We remark that if $(X, d)$ is not compact, then there do exist functions in $\operatorname{Lip}(\Sigma, \sigma)$ which are not continuous in the Gelfand topology. One such function $g$ on $\Sigma$ is

$$
g(\varphi)=\sigma(\varphi, X)=\inf \{\sigma(\varphi, x): x \in X\} \quad \varphi \in \Sigma .
$$

It is readily checked that $g$ is a bounded Lipschitz function on $(\Sigma, \sigma)$. Since $X$ is a proper closed subset of $(\Sigma, \sigma)$ by Theorem 3.9 and Lemma 3.8, $g$ is not identically zero. But since $X$ is dense in $\Sigma$ in the Gelfand topology and $g=0$ on $X$, we see that $g$ cannot be continuous on $\Sigma$ in the Gelfand topology. Hence there can be no $f \in \operatorname{Lip}(X, d)$ such that $g=\hat{f}$. Thus for non-compact $(X, d)$ the Gelfand mapping does not take $\operatorname{Lip}(X, d)$ onto $\operatorname{Lip}(\Sigma, \sigma)$.

The Banach algebra $A$ is called regular if for each proper subset $K$ of $\Sigma$ closed in the Gelfand topology and each point $\varphi \in \Sigma-K$, there exists an $f \in A$ such that $\hat{f}(\varphi)=1$ and $\hat{f}(K)=0$. 
That $\operatorname{Lip}(X, d)$ is regular follows from the fact that a Lipschitz condition is preserved under truncation. The following proposition, due to J. Lindberg, shows that such algebras are regular in general. Call an algebra of functions $A$ on a space $X$ closed under truncation if the function $\min (f, 1)$ belongs to $A$ for all real-valued $f \in A$.

Proposition 4.2. Let $X$ be a compact Hausdorff space and $A$ be a self-adjoint subalgebra of $C(X)$ which separates the points of $X$ and contains the constant functions. If $A$ is closed under truncation, then $A$ is regular.

Proof. Let $x \in X$ and let $V$ be a neighborhood of $x$. Choose $f \in C(X)$ such that $f(x)=0, f(X-V)=3 / 2$ and $0 \leqq f \leqq 3 / 2$. Since $A$ is dense in $C(X)$, there exists $g \in A$ with $\|f-g\|_{\infty}<1 / 2$; we may take $g$ to be real-valued. Then $g \geqq 1$ on $X-V$. Let $h=\min (g, 1)$, iso that $h=1$ on $X-V$. Also, $|h(x)|<1 / 2$. Set

$$
f=(h-1) /(h(x)-1) \text {. }
$$

Then $f \in A$ and $f(x)=1$ while $f(X-V)=0$. Thus $A$ is regular.

Corollary 4.3. $\operatorname{Lip}(X, d)$ is regular.

Proof. Let $f \in \operatorname{Lip}(X, d)$ be real and set $T f=\min (f, 1)$. Then

$$
|(T f)(x)-(T f)(y)| \leqq|f(x)-f(y)| \quad x, y \in X
$$

which may be seen by comparing the graphs of $f$ and $T f$, or by checking each of the possible cases for a given $x$ and $y$. It is immediate from this that $\operatorname{Lip}(X, d)$ is closed under truncation.

Also $\operatorname{Lip}(X, d)$ is self-adjoint, point-separating and contains the constants. Since $X$ is dense in $\Sigma$ in the Gelfand topology, it follows that $\operatorname{Lip}(X, d)$ is closed under truncation if and only if $\{\hat{f}: f \in \operatorname{Lip}(X, d)\}$ is closed under truncation. Hence, $\operatorname{Lip}(X, d)$ is regular.

5. We now consider the problem of describing the homomorphisms from one Lipschitz algebra into another. For Lipschitz algebras on compact metric spaces we are able to identify the homomorphisms and the description is given in the proposition below. We first make a few general comments on homomorphisms of Banach algebras. To avoid technical trivialities we shall always assume that homomorphisms carry the identity of one algebra into the identity of the other.

It is well known [2] that if $T$ is a homomorphism of a semisimple commutative Banach algebra $A_{1}$ into another $A_{2}$, then $T$ is automatically continuous and induces a dual mapping $\tau: \Sigma_{2} \rightarrow \Sigma_{1}$ of 
the carrier spaces. This dual mapping $\tau$ is defined as follows : given $\varphi \in \Sigma_{2}$ define the multiplicative linear functional $\tau^{\varphi} \in \Sigma_{1}$ by

$$
(\tau \varphi)(f)=\varphi(T f) \quad f \in A_{1} .
$$

The homomorphism $T$ is in turn induced by the mapping $\tau$ by means of (5.1). The dual mapping $\tau$ is always continuous. If $T$ maps $A_{1}$ onto $A_{2}$, then $\tau$ is one-to-one; if $\tau$ is onto, then $T$ is one-to-one. If $T$ is an isomorphism of $A_{1}$ onto $A_{2}$, then the dual mapping is a homeomorphism of $\Sigma_{2}$ onto $\Sigma_{1}$. For a detailed discussion see [2, p. 75, p. 116].

Now let $\left(X_{1}, d_{1}\right)$ and $\left(X_{2}, d_{2}\right)$ be compact metric spaces and let $A_{1}=\operatorname{Lip}\left(X_{1}, d_{1}\right)$ and $A_{1}=\operatorname{Lip}\left(X_{2}, d_{2}\right)$. By Theorem 3.9 the carrier spaces of $A_{1}$ and $A_{2}$ are $X_{1}$ and $X_{2}$ respectively. Let $T$ be a homomorphism of $A_{1}$ into $A_{2}$. Then the dual mapping $\tau$ takes $X_{2}$ into $X_{1}$ and equation (5.1) can be written

$$
f(\tau x)=(T f)(x) \quad f \in A_{1}, x \in X_{2} .
$$

The converse situation does not hold in general. That is, given a continuous mapping $\tau: X_{2} \rightarrow X_{1}$, the mapping $T$ defined on $A_{1}$ by (5.2) will not in general take $A_{1}$ into $A_{2}$. The following proposition identifies those mappings $\tau: X_{2} \rightarrow X_{1}$ which are dual to homomorphisms of the Lipschitz algebras.

THEOREM 5.1. Let $A_{i}=\operatorname{Lip}\left(X_{i}, d_{i}\right)$ where $\left(X_{i}, d_{i}\right)$ is compact, $i=1,2$. Then every homomorphism $T: A_{1} \rightarrow A_{2}$ is of the form

$$
(T f)(x)=f(\tau x) \quad f \in A_{1}, x \in X_{2}
$$

where $\tau: X_{2} \rightarrow X_{1}$ satisfies

$$
d_{1}(\tau x, \tau y) \leqq K d_{2}(x, y) \quad x, y \in X_{2}
$$

for some positive constant $K$. Conversely, if $T$ is defined on $A_{1}$ by (5.3) where $\tau: X_{2} \rightarrow X_{1}$ satisfies (5.4), then $T$ is a homomorphism of $A_{1}$ into $A_{2} . \quad T$ is one-to-one if and only if $\tau\left(X_{2}\right)=X_{1} . \quad T$ takes $A_{1}$ onto $A_{2}$ if and only if $\tau$ satisfies the additional condition

$$
K^{\prime} d_{2}(x, y) \leqq d_{1}(\tau x, \tau y) \quad x, y \in X_{2} .
$$

for some positive constant $K^{\prime}$.

Proof. Suppose $T: A_{1} \rightarrow A_{2}$ is a homomorphism with dual mapping $\tau: X_{2} \rightarrow X_{1}$. For each $s \in X_{1}$ define the function $f_{s}$ on $X_{1}$ by $f_{s}(t)=$ $d_{1}(t, s), t \in X_{1}$. Then $f_{s} \in A_{1}$ and

$$
\left\|f_{s}\right\|_{\infty}+\left\|f_{s}\right\|_{d_{1}} \leqq \operatorname{diameter}\left(X_{1}, d_{1}\right)+1
$$


for all $s \in X_{1}$. Thus the set $\left\{f_{s}: s \in X_{1}\right\}$ is bounded in $A_{1}$. Since $T$ is continuous, the set $\left\{T f_{s}: s \in X_{1}\right\}$ is bounded in $A_{2}$. Then there exists a positive constant $K$ such that $\left\|T f_{s}\right\|_{d_{2}} \leqq K$, all $s \in X_{1}$. Thus for all points $x, y \in X_{2}$ and $s \in X_{1}$ we have

$$
\frac{\left|\left(T f_{s}\right)(x)-\left(T f_{s}\right)(y)\right|}{d_{2}(x, y)}=\frac{\left|d_{1}(s, \tau x)-d_{1}(s, \tau y)\right|}{d_{2}(x, y)} \leqq K .
$$

Taking $s=\tau y$ in this inequality, we obtain

$$
d_{1}(\tau x, \tau y) \leqq K d_{2}(x, y)
$$

for all $x, y \in X_{2}$. Hence, the mapping $\tau$ satisfies (5.4).

Conversely, if $T$ is defined by (5.3) in terms of a $\tau$ satisfying (5.4), then

$$
\begin{aligned}
\|T f\|_{d_{2}} & =\sup \left\{\frac{|(T f)(x)-(T f)(y)|}{d_{2}(x, y)}: x, y \in X_{2}\right\} \\
& =\sup \left\{\frac{|f(\tau x)-f(\tau y)|}{d_{2}(x, y)}: x, y \in X_{2}\right\} \\
& \leqq K \sup \left\{\frac{|f(\tau x)-f(\tau y)|}{d_{1}(\tau x, \tau y)}: x, y \in X_{2}\right\} \\
& \leqq K\|f\|_{d_{1} .}
\end{aligned}
$$

Also,

$$
\begin{aligned}
\|T f\|_{\infty} & =\sup \left\{|f(\tau x)|: x \in X_{2}\right\} \\
& \leqq \sup \left\{|f(y)|: y \in X_{1}\right\}=\|f\|_{\infty} .
\end{aligned}
$$

Hence $T$ carries $A_{1}$ into $A_{2}$ and it is easily seen to be a homomorphism.

We know in general that if $\tau$ is onto, then $T$ is one-to-one. Suppose $T$ is one-to-one. If $\tau$ is not onto, then there exists $s \in X_{1}$ with $s \notin \tau\left(X_{2}\right)$. The continuity of $\tau$ implies that $\tau\left(X_{2}\right)$ is compact since $\left(X_{2}, d_{2}\right)$ is compact. But then by the regularity of $A_{1}$ we can choose $f \in A_{1}$ with $f(s)=1$ and $f=0$ on $\tau\left(X_{2}\right)$. Then $(T f)(x)=f(\tau x)=0$, all $x \in X_{2}$. This contradicts the fact that $T$ is one-to-one. Hence, $\tau$ is onto.

Suppose now that $T$ is onto. Then $\tau$ is one-to-one and we can define a new metric $d^{\prime}$ on $X_{2}$ by

$$
d^{\prime}(x, y)=d_{1}(\tau x, \tau y) \quad x, y \in X_{2} .
$$

Let $A^{\prime}=\operatorname{Lip}\left(X_{2}, d^{\prime}\right)$. Let $f \in A_{2}$ and choose $g \in A_{1}$ such that $T g=f$. Then since

$$
\frac{|f(x)-f(y)|}{d^{\prime}(x, y)}=\frac{|g(\tau x)-g(\tau y)|}{d_{1}(\tau x, \tau y)}
$$


for all $x, y \in X_{2}$, we see that $\|f\|_{d^{\prime}} \leqq\|g\|_{a_{1}}$. Hence $A_{2} \subset A^{\prime}$. In particular, the function $f$ defined by $f(x)=d_{2}(x, u), x \in X_{2}$, belongs: to $A^{\prime}$ for each $u \in X_{2}$. It follows that

$$
d_{2}(x, y) \leqq K^{\prime \prime} d^{\prime}(x, y) \quad x, y \in X_{2}
$$

for some constant $K^{\prime \prime}$. This yields (5.5) with $K^{\prime}=1 / K^{\prime \prime}$.

Finally suppose that $\tau$ satisfies (5.5) in addition to (5.4). Then $\tau$ is one-to-one and the metric $d^{\prime}$ on $X_{2}$ defined by (5.6) is boundedly equivalent to the metric $d_{2}$. By Corollary 3.5, $A_{2}$ and $A^{\prime}$ must have the same elements. But if $f \in A^{\prime}$, then the function $g$ defined on $X_{1}$ by $g(s)=f\left(\tau^{-1} s\right), s \in X_{1}$, belongs to $A_{1}$ and $T g=f$. Thus $T$ maps $A_{1}$ onto $A^{\prime}$, and hence onto $A_{2}$. This completes the proof.

CoRollaRY 5.2. Every automorphism $T$ of $\operatorname{Lip}(X, d)$ where $(X, d)$ is compact is of the form

$$
(T f)(x)=f(\tau x) \quad f \in \operatorname{Lip}(X, d), x \in X
$$

where $\tau: X \rightarrow X$ is a homeomorphism satisfying

$$
K_{1} d(x, y) \leqq d(\tau x, \tau y) \leqq K_{2} d(x, y) \quad x, y \in X
$$

for some positive constants $K_{1}$ and $K_{2}$.

\section{REFERENCES}

1. Loomis, Abstract Harmonic Analysis, Van Nostrand, New York (1953).

2. C. Rickart, General Theory of Banach Algebras, Van Nostrand, New York (1960). 



\section{PACIFIC JOURNAL OF MATHEMATICS}

\section{EDITORS}

RalPh S. Phillips

Stanford University

Stanford, California

M. G. Arsove

University of Washington

Seattle 5 , Washington
J. Dugundu

University of Southern California

Los Angeles 7, California

Lowell J. Paige

University of California

Los Angeles 24, California

\section{ASSOCIATE EDITORS}
E. F. BECKENBACH
D. DERRY
H. L. ROYDEN
E. G. STRAUS
T. M. CHERRY
M. OHTSUKA
E. SPANIER
F. WOLF

\section{SUPPORTING INSTITUTIONS}

\author{
UNIVERSITY OF BRITISH COLUMBIA \\ CALIFORNIA INSTITUTE OF TECHNOLOGY \\ UNIVERSITY OF CALIFORNIA \\ MONTANA STATE UNIVERSITY \\ UNIVERSITY OF NEVADA \\ NEW MEXICO STATE UNIVERSITY \\ OREGON STATE UNIVERSITY \\ UNIVERSITY OF OREGON \\ OSAKA UNIVERSITY \\ UNIVERSITY OF SOUTHERN CALIFORNIA
}

\author{
STANFORD UNIVERSITY \\ UNIVERSITY OF TOKYO \\ UNIVERSITY OF UTAH \\ WASHINGTON STATE UNIVERSITY \\ UNIVERSITY OF WASHINGTON \\ AMERICAN MATHEMATICAL SOCIETY \\ CALIFORNIA RESEARCH CORPORATION \\ SPACE TECHNOLOGY LABORATORIES \\ NAVAL ORDNANCE TEST STATION
}

Mathematical papers intended for publication in the Pacific Journal of Mathematrcs should be typewritten (double spaced), and the author should keep a complete copy. Manuscripts may be sent to any one of the four editors. All other communications to the editors should be addressed to the managing editor, L. J. Paige at the University of California, Los Angeles 24, California.

50 reprints per author of each article are furnished free of charge; additional copses may be obtained at cost in multiples of 50 .

The Pacific Journal of Mathematics is published quarterly, in March, June, September, and December. Effective with Volume 13 the price per volume (4 numbers) is $\$ 18.00$; single issues, $\$ 5.00$. Special price for current issues to individual faculty members of supporting institutions and to individual members of the American Mathematical Society: $\$ 8.00$ per volume; single issues $\$ 2.50$. Back numbers are available.

Subscriptions, orders for back numbers, and changes of address should be sent to Pacific Journal of Mathematics, 103 Highland Boulevard, Berkeley 8, California.

Printed at Kokusai Bunken Insatsusha (International Academic Printing Co., Ltd.), No. 6 , 2-chome, Fujimi-cho, Chiyoda-ku, Tokyo, Japan.

\section{PUBLISHED BY PACIFIC JOURNAL OF MATHEMATICS, A NON-PROFIT CORPORATION}

The Supporting Institutions listed above contribute to the cost of publication of this Journal, but they are not owners or publishers and have no responsibility for its content or policies. 


\section{Pacific Journal of Mathematics}

\section{Vol. 13, No. $4 \quad$ June, 1963}

Dallas O. Banks, Bounds for eigenvalues and generalized convexity ........... 1031

Jerrold William Bebernes, A subfunction approach to a boundary value problem for

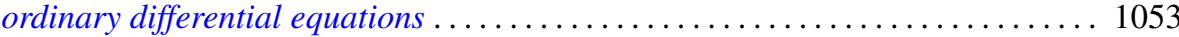

Woodrow Wilson Bledsoe and A. P. Morse, A topological measure construction . . . 1067

George Clements, Entropies of several sets of real valued functions . . . . . . . . . 1085

Sandra Barkdull Cleveland, Homomorphisms of non-commutative *-algebras . . . . . 1097

William John Andrew Culmer and William Ashton Harris, Convergent solutions of

ordinary linear homogeneous difference equations . . . . . . . . . . . . . . . 1111

Ralph DeMarr, Common fixed points for commuting contraction mappings . . . . . . 1139

James Robert Dorroh, Integral equations in normed abelian groups . . . . . . . . 1143

Adriano Mario Garsia, Entropy and singularity of infinite convolutions . . . . . . . 1159

J. J. Gergen, Francis G. Dressel and Wilbur Hallan Purcell, Jr., Convergence of extended Bernstein polynomials in the complex plane ................. 1171

Irving Leonard Glicksberg, A remark on analyticity of function algebras . . . . . . 1181

Charles John August Halberg, Jr., Semigroups of matrices defining linked operators

with different spectra ................................. 1187

Philip Hartman and Nelson Onuchic, On the asymptotic integration of ordinary

differential equations . . . . . . . . . . . . . . . . . . . . . . . . . . . . 1193

Isidore Heller, On a class of equivalent systems of linear inequalities . . . . . . . . . 1209

Joseph Hersch, The method of interior parallels applied to polygonal or multiply

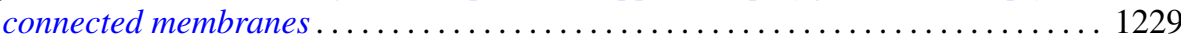

Hans F. Weinberger, An effectless cutting of a vibrating membrane . . . . . . . . . . 1239

Melvin F. Janowitz, Quantifiers and orthomodular lattices ....

Samuel Karlin and Albert Boris J. Novikoff, Generalized convex inequalities . .

Tilla Weinstein, Another conformal structure on immersed surfaces of negative

curvature.

Gregers Louis Krabbe, Spectral permanence of scalar operators

Shige Toshi Kuroda, Finite-dimensional perturbation and a representaion of

scattering operator.

Marvin David Marcus and Afton Herbert Cayford, Equality in certain

inequalities

Joseph Martin, A note on uncountably many disks .

Eugene Kay McLachlan, Extremal elements of the convex cone of semi-norms . . . . 1335

John W. Moon, An extension of Landau's theorem on tournaments . .

Louis Joel Mordell, On the integer solutions of $y(y+1)=x(x$

Kenneth Roy Mount, Some remarks on Fitting's invariants .....

Miroslav Novotný, Über Abbildungen von Mengen ............

Robert Dean Ryan, Conjugate functions in Orlicz spaces.

John Vincent Ryff, On the representation of doubly stochastic operators . . . . . . . . 1379

Donald Ray Sherbert, Banach algebras of Lipschitz functions .

James McLean Sloss, Reflection of biharmonic functions across analytic boundary

conditions with examples.

L. Bruce Treybig, Concerning homogeneity in totally ordered, connected topological space....

John Wermer, The space of real parts of a function algebra...

James Juei-Chin Yeh, Orthogonal developments of functionals and related theorems

in the Wiener space of functions of two variables......... 